\title{
METAL-POLYMER-VACUUM INTERFACE FORMATION IN THE HIGH STATIC ELECTRIC FIELD
}

\author{
A. KarpowicZ \\ Institute of Electrical Engineering and Technology, Wroclaw Technical University \\ pl. Grunwaldzki 13, 50-377. Wroclaw, Poland \\ AND M. RADNY \\ Institute of Experimental Physics, University of Wroclaw, \\ Cybulskiego 36, 50-205 Wroclaw, Poland \\ (Received June 14, 1991; in final form September 9, 1991)
}

\begin{abstract}
A qualitative discussion of the simple interface barrier defect-type model (Bardeen-like) trying to explain the metal-polymer-vacuum interface formation in the high static elcctric field (field emission microscopy) is presented.
\end{abstract}

PACS numbers: $73.40 . \mathrm{Gk}, 73.40 . \mathrm{Ns}, 79.70 .+\mathrm{q}$

\section{Introduction}

The phenomenon of an electron emission from cold metals in high static electric fields was observed for the first time by Lilienfeld in 1922 [1]. Six years later Fowler and Nordheim [2] derived their well-known formula for the field emission current analyzing the electron tumnelling through the potential barrier.

In order to understand the phenomenon of an electron transport through the interface between two solids (metal-metal, metal-semiconductor, etc.) the idea of interband tunnelling was proposed by Zener [3]. According to him interband tunnelling should occur between a filled band to a next higher unfilled band by the application of the electric field. In this model the energy gap in the electronic band structure of the solid could be treated in the manner of a potential barrier called usually the Schottky barrier [4]. Its height is simply the difference between the metal Fermi level and the bottom of the conduction band of the semiconductor (insulator). In general, the formation of the interface potential barrier is strongly determined by the position of the Fermi level at the contact. Bardeen [5] was the first who pointed out the significant role of the intrinsic electronic surface states (gap states) in the stabilization (pinning) process of the Fermi level at such interfaces (Bardeen model [6]). 
Due to of the great importance of the character of the interface potential barrier in solid state devices, extensive studies of its formation are carried out for many systems. In this paper, where we focus our attention on the process of interface formation between the metal and a polyethylene film in the field emission experiment (metal-polymer-vacuum system) [7-10]. In the following paper [18] we discuss a phenomenon of a negative resistance in the aluminum-plasma-polymerized polystyrene (p-p PS)-aluminium junction.

A systematic studies of the field electron emission properties of a dielectric coated metal (tungsten) has been reported by Latham and Mousa [7, 8] and Karpowicz and Surma [9, 10]. In both experiments, among other unusual properties, the field emission was observed only after a switch-on voltage has been applied to the sample. Then the electron tunnelling could continue at much lower voltages. The qualitative explanation of this effect was done on the base of the hot electron emission model $[7,8]$. It is our aim here to present an alternative physical picture of this phenomenon. Our discussion is based on the general properties of the "optical" potential and on the recent electronic structure calculations performed for polymers [11] (Section 3).

\section{Experimental results}

The apparatus and the method of the deposition of polyethylene (PE) onto a tungsten tip were described in $[9,10,14]$. In brief, the experimental results can be summarized as follows:

i) Switch-on effect.

For a virgin sample when the applied voltage is slowly increasing, at a point $U_{\mathbf{S}}$ (corresponding to the field $1-3.5 \times 10^{9} \mathrm{~V} / \mathrm{m}$ ) the emission current suddenly "switches-on" from an eflective zero value to a saturated value $\left(1-5 \times 10^{-6} \mathrm{~A}\right)$. Consequently, no image appears up to this stimulated voltage (curve 1 in Fig. 1). With

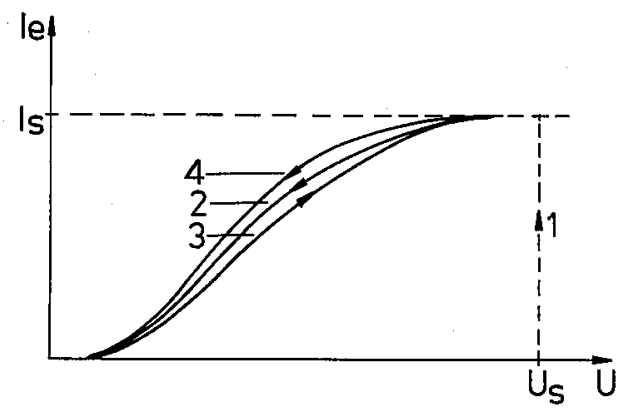

Fig. 1. Field emission current-voltage $(I-V)$ characteristics of polyethylene-tungsten system.

decreasing of the applied voltage the value of the emision current falls smoothly (curve 2 in Fig. 1) to the effective zero value. ii) IIysteresis effect. 
After switch-on eflect occurred the subsequent attempts of decreasing and increasing of the applied voltage the $I-V$ characteristic follows the curve 3 and 4 , i.e. on subsequently re-cycling the voltage the characteristic exhibits a hysteresis. iii) Saturation effect.

An anomalously wide range of field emission voltages and saturation of the field emission current value for higher voltages was observed. IIence, only in the low-field region of the $I-V$ data a linear Fowler-Nordheim characteristic is exhibited.

Field emission experiments had to be restricted because of the fast rise in the ambient pressure during the course of microscopy, which was associated with sudden changes in the geometry of the deposit and a high instability of the emission current. FEM patterns showed a spot or two of bright emission sometimes with a dynamic structure within the spot or, alternatively, a large area of a strong and relatively uniform emission.

\section{Discussion}

Some of the emission properties of the metal-polymer system are similar to those observed for the other metal-semiconductor junctions. Namely, in both systems the phenomena of the saturation [15] and hysteresis [16] of the field emission current were observed. The saturation effect can be understood as the generation rate of mobile carriers throughout the whole depth of the sample [17], while the hysteresis can be caused by the internal electric field in the semiconductor arising from the charging of the deep localized electron states [17].

There is, however, one important difference between the semiconductor-metal and polyethylene-tungsten junction discussed here. In the last case, the switch-on effect is observed for the certain value $\left(U_{\mathrm{S}}\right)$ of the applied voltage (curve 1 in Fig. 1). In general, this effect indicates that the inelastic electron tunnelling occurs in the system and in principle one can try to find the finite structure of it. However, no interaction of the tunnelling electrons with phonons, photons, plasmons, vibration modes etc., have been identified from the experimental data analysis [7-11].

From the theoretical point of view, an application of the classical picture of the electron tunnelling process to explain the switch-on effect is not satisfactory. The quasi-classical expression for the tunnelling current per unit area calculated for the real part of the model potential (Fig. 2) of the metal-polymer interface can be written as

$$
j \approx \int D(E) f(E) v \mathrm{~d} k
$$

where $E$ is an electron kinetic energy associated with the normal momentum component, $f(E)$ is the Fermi distribution function, and $v$ is the velocity of the incident electron associated with a state of a wave number $k$. The function $D(E)$ in Eq. (1) is the transmission coefficient. The most common approximation for $D(E)$ is the expression obtained from the WKB (Wentzel-Kramers-Brillouin) method

$$
D(E)=\exp \left(-2 \int_{x_{1}}^{x_{2}}[2 m(V-E)]^{1 / 2} \mathrm{~d} x\right),
$$




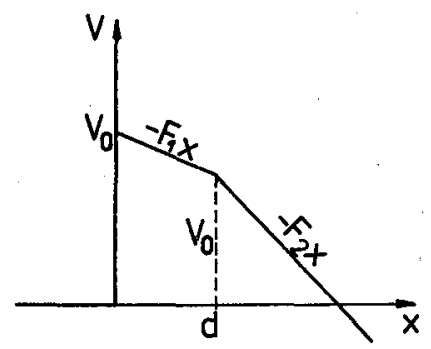

Fig. 2. Real part of the model potential barrier of metal-insulator-vacuum system (see text, Eq. (A1)).

where $x_{1}, x_{2}$ are the classical turning points of the electron of the energy $E$. In this treatment the function $D(E)$ and then the tunnelling current depend only on the height and width of the barrier. Within this model, by modulation of the internal field $F_{1}$, one can roughly describe the hysteresis effect which occurs after the switch-on effect (Fig. 1). However, below the threshold (switch-on) voltage the transmission coefficient $D(E)$ ought to be close to zero (no emission current), i.e. the width or hight of the real potential barrier should go to infinity (Eqs. (1) and (2)).

The introduction of a small negative (or positive) imaginary part to the real potential barrier ( $W_{0}$ in Eq. ( $\left.A 1\right)$, see Fig. 1) changes the classical picture of the tunnelling process drastically. The analytical expressions for the density of the field emission current calculated from Eq. $(\Lambda 1)$ are presented in the Apendix. Although performed for the more complex and interesting situation, numerical results qualitatively agree with the results of Ref. [12]. Ilowever, the physics underlying the described phenomenon provides directly the new model of the tunnelling process in the polymers. In order to illustrate it, the general ideas of the "optical potential" treatment is presented below. Following [12] let us consider the rectangular potential barrier of the height $V_{0}$ and width $b$, where

$$
V= \begin{cases}V_{0} \mp \mathrm{i} W_{0}, & 0<x<b \\ 0 & \text { otherwise. }\end{cases}
$$

Hence, the wave function of the electron inside the barrier can be written in the form [12]

$$
\Psi=A \exp \left(-k_{1} x \mp \mathrm{i} k_{2} x\right),
$$

where

$$
\begin{aligned}
& k_{1}=R \cos (\alpha / 2), \\
& k_{2}=R \sin (\alpha / 2)
\end{aligned}
$$

and

$$
R=\left\{2\left(V_{0}-E\right)^{1 / 2}\left[1+\left(\frac{W_{0}}{V-E}\right)^{1 / 4}\right]\right\}^{1 / 2}
$$




$$
\alpha=\tan ^{-1}\left[W_{0} /(V-E)\right] .
$$

Let us notice that the electron wave function inside the barrier (4) consists of two parts: attenuating $\left(\mathrm{e}^{-k_{1} x}\right)$ and running one $\left(\mathrm{e}^{ \pm \mathrm{i} k_{2} x}\right)$. When the imaginary part $W_{0}$ in the potential (3) is negative the oscillating phase in Eq. (4) $\left(\mathrm{i} k_{2} x\right)$ superimposes on the attenuating one $\left(-k_{1} x\right)$ and the electron is "scattered forward" $(x>0$, see Fig. 3). As the result, the field emission current - calculated from Eq. (A21) in the Appendix - increases. In the opposite case, when $W_{0}$ becomes positive $(>0)$ the term $\left(\mathrm{e}^{-\mathrm{i} k_{2} x}\right)$ represents now the "backward scattering", so that the transmission probability of the electron through the barrier is strongly reduced and the field emission current falls off.

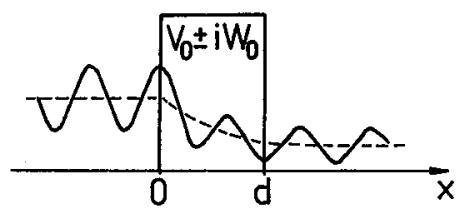

Fig. 3. Rectangular potential barricr with an "optical" part $W_{0}$. The wave function inside the barrier is dumping for real potential ( $W_{0}=0$, broken curve), while oscillations are due to the running term in the clectron wave function inside the barrier $\left(W_{0} \neq 0\right)$.

At this point we would like to mention calculations of the electronic structure of the polymers performed recently within the Coherent Potential Approximation [11]. An influence of disorder effects on the polymer electronic structure. (trans-polyacetylene in [11]) has been investigated starting with the ideas of two kind of impurities which can be responsible for the electron conductivity properties of such media. One is the site-type impurity, for which the site energy of the electrons is locally modulated around the charged impurity. In this case, with increasing concentration of the impurities the reduction of the energy gap takes place, i.e. the new localized electronic states in the gap and beyond the band edges can be observed. The other type is the bond-type impurity. One important example of this kind of defect would be the change in the distance between neighbouring $\mathrm{CII}$ unit due to the inhomogeneous distribution of the charged impurities. With increasing concentration of the bond-type impurities - in contrast to the situation analyzed before - the band gap increases and no localized states can be observed in it. It seems to be reasonable that in the case of the metal-polymer-vacuum junction the impurities of the bond-type give rise to the forward scattering $\left(W_{0}<0\right)$, while the site-type impurities to backward scattering $\left(W_{0}>0\right)$, for the tunnelling electrons. Hence, the qualitative physical picture of the switch-on mechanism in the metal-polymer-vacuum system in the high static electric field can be seen as follows.

When the external electric field is lower than the switch-on value, the electrons injected into polyethylene from the field emitter are likely trapped in the deep localized deffect states in the electronic structure of the polymer (backward 
scattering - no tunnelling current - curve 1 in Fig. 1). This process leads to the creation of the space charge in the polymer and shifts up the Fermi level in the polyethylene film towards its conduction band edge reducing the distance (in energy) between them. With further increasing of the applied voltage the Fermi level crosses finally the edge of the polymer conduction band and the switch-on effect of the field emission current is observed (forward scattering - curve 1 in Fig. 1). Because in the subsequent observations (curves 2, 3, 4 in Fig. 1) the switch-on effect does not appear (curve 3), it is reasonable to state that for this value of the electric field the localized charged electron states "pin" the Fermi level at a position near the polyethylene conduction band. At this stage of the tunnelling process, electrons tunnel into the conduction band (Zener-like effect) or into a band formed near to it (curve 2, 3 and 4 in Fig. 1), independently of the value of the applied electric field.

\section{Summary}

It is evident from the experimental data that the electronic properties of the polymer are in striking contrast to those of the semiconductors. The mechanism of the hot electron emission for the switch-on effect in the field emission experiment proposed by Latham and Mousa [7,8] neglects this fact and the phenomenon is explained via the existence of the hot electrons at the insulator-vacuum interface. Some of these electrons can be thermionically emitted over the surface potential barrier. We want to point out that the explanation of the switch-on effect discussed here is based on the recent polymer band structure predictions and on the old "defect"-type model of the interface potential barrier formation (Bardeen-like, [6]). The main assumption of the model presented by us is that the deposition of the insulator on the metal surface induces (or conserves the existing) deep (in energy) defect electron states that can pin the metal Fermi energy after applying the appropriate (switch-on) value of the external field.

\section{Appendix}

The complex potential barrier model of the metal-insulator-vacuum system under an applied electric field adopted in this part of the work has the form

$$
V(x)=\left\{\begin{array}{lll}
0 & \text { for } x<0 \\
V_{0} \pm \mathrm{i} W_{0} & \text { for } 0<x<d, & \text { where } V_{0}=E_{\mathrm{F}}+\varphi-F_{1} x, \\
V_{0}^{\prime} \pm \mathrm{i} W_{0}^{\prime} & \text { for } x>d, & \text { where } V_{0}^{\prime}=V_{0}-F_{2} x
\end{array}\right.
$$

where $d$ is the width of the barrier of the metal-insulator junction, $F_{1}$ is an electric field in the insulator, $F_{2}$ is an applied electric field strength, $V_{0}$ and $V_{0}^{\prime}$ are heights of the barrier at the metal-insulator and insulator-vacuum interfaces, and $W_{0}$ and $W_{0}^{\prime}$ are imaginary parts of these potentials (Hertree atomic units are used throught). In this three regions (see Fig. 3.), the solutions of the Schroedinger equation are as follows:

$$
\psi_{1}=\exp (\mathrm{i} k x)+B \exp (-\mathrm{i} k x), \quad x<0,
$$




$$
\begin{aligned}
& \psi_{2}=\beta_{1}[\operatorname{Bi}(-z)+\mathrm{i} A \mathrm{i}(-z)]+\beta_{2}[\mathrm{Bi}(-z)+\mathrm{i} \mathrm{Ai}(-z)], \quad 0<x<d, \\
& \psi_{3}=C[\operatorname{Bi}(-z)+\mathrm{i} A \mathrm{i}(-z)], \quad x>d,
\end{aligned}
$$

where $\mathrm{i}=\sqrt{-1}, k=\sqrt{2 E}$, and $\mathrm{Bi}(-z)$ and $\Lambda \mathrm{i}(-z)$ are independent solutions of the wave equation for the linear potential [13] known as Airy functions. Their linear combinations (as in Eq. (A3)) are also independent solutions of the same wave equation. In the case discussed here arguments of Airy functions are negative, so that $\mathrm{Ai}(z)$ and $\mathrm{Bi}(z)$ oscillate and their asymptotic forms are simple

$$
\begin{aligned}
& \operatorname{Ai}(-z) \approx \pi^{-1 / 2} z^{-1 / 4} \sin (s+\pi / 4), \\
& B i(-z) \approx \pi^{-1 / 2} z^{-1 / 4} \cos (s+\pi / 4),
\end{aligned}
$$

where

$$
\begin{aligned}
& z(x)=2\left(E-V_{0}^{\prime} \pm \mathrm{i} W_{0}^{\prime}\right) / \alpha_{1}^{2}-\alpha_{1} x \quad \text { for } \quad 0 \leq x \leq d, \\
& z(x)=2\left(E-V_{0} \pm \mathrm{i} W_{0}\right) / \alpha_{2}^{2}-\alpha_{2} x \quad \text { for } \quad x \geq d, \\
& \alpha_{1}=\sqrt[3]{2 F_{1}}, \quad \alpha_{2}=\sqrt[3]{2 F_{2}} \text { and } s=2 / 3 z^{-3 / 2} .
\end{aligned}
$$

It is easy to see from Eqs. (A5) and (A6) that the wave function in Eq. (A4) represents an electron travelling to $-\infty$ (Fig. 2) after tunnelling. In this way we have introduced the outgoing boundary condition in our system.

The transmission probability for our solvable one-dimensional Schroedinger equation is calculated imposing continuity of $\psi_{i}$ and $\psi_{i}^{\prime}(i=1,2,3)$ at $x=0$ and $x=d$

$$
\begin{aligned}
& \left.\frac{\psi_{1}^{\prime}}{\psi_{1}}\right|_{x=0}=\left.\frac{\psi_{2}^{\prime}}{\psi_{2}}\right|_{x=0} \\
& \left.\frac{\psi_{2}^{\prime}}{\psi_{2}}\right|_{x=d}=\left.\frac{\psi_{3}^{\prime}}{\psi_{3}}\right|_{x=d} .
\end{aligned}
$$

This procedure gives a set of linear equations for the coefficients of the eigenfunctions (A2) of the one-electron Schroedinger equation

$$
\begin{aligned}
& 1+B=\left(\pi^{2} z_{0}\right)^{-1 / 4}\left\{\beta_{1} \exp \left[\mathrm{i}\left(s_{0}+\pi / 4\right)\right]+\beta_{2} \exp \left[-\mathrm{i}\left(s_{0}+\pi / 4\right)\right]\right\} \\
& \frac{k(1-B)}{1+B}=\alpha_{1} z_{0}^{1 / 2} \frac{\beta_{1} \exp \left[\mathrm{i}\left(s_{0}+\pi / 4\right)\right]-\beta_{2} \exp \left[-\mathrm{i}\left(s_{0}+\pi / 4\right)\right]}{\beta_{1} \exp \left[\mathrm{i}\left(s_{0}+\pi / 4\right)\right]+\beta_{2} \exp \left[-\mathrm{i}\left(s_{0}+\pi / 4\right)\right]} \\
& C z_{D}^{-1 / 4} \exp \left[\mathrm{i}\left(s_{D}+\pi / 4\right)\right] \\
& =z_{d}^{-1 / 4}\left\{\beta_{1} \exp \left[\mathrm{i}\left(s_{d}+\pi / 4\right)\right]+\beta_{2} \exp \left[-\mathrm{i}\left(s_{\mathrm{d}}+\pi / 4\right)\right]\right\} \\
& \alpha_{2} z_{D}^{1 / 2}=\alpha_{1} z_{d}^{1 / 2} \frac{\beta_{1} \exp \left[\mathrm{i}\left(s_{d}+\pi / 4\right)\right]-\beta_{2} \exp \left[-\mathrm{i}\left(s_{d}+\pi / 4\right)\right]}{\beta_{1} \exp \left[\mathrm{i}\left(s_{d}+\pi / 4\right)\right]+\beta_{2} \exp \left[-\mathrm{i}\left(s_{d}+\pi / 4\right)\right]}
\end{aligned}
$$


where

$$
\begin{aligned}
z_{0}=z_{1}(0), & s_{0}=2 / 3 z_{0}^{3 / 2}, & z_{d}=z_{1}(d), \\
s_{d}=2 / 3 z_{d}^{3 / 2}, & z_{D}=z_{2}(d), & s_{D}=2 / 3 z_{D}^{3 / 2} .
\end{aligned}
$$

The above equations are solved with respect to $C$ yielding the transmission probability in the form

$$
C=\left(\pi^{2} z_{d}\right)^{-1 / 4}\left(z_{0} z_{D}\right)^{1 / 4}(B+1) \beta \exp \left[-\mathrm{i}\left(s_{D}+\pi / 4\right)\right]
$$

where

$$
\begin{aligned}
& \beta=\frac{\exp \left[-\mathrm{i}\left(s_{0}+\pi / 4\right)\right] \exp \left[\mathrm{i}\left(s_{d}+\pi / 4\right)\right]+A \exp \left[-2 \mathrm{i}\left(s_{d}+\pi / 4\right)\right] \exp \left(-\mathrm{i} s_{1}\right)}{1+A \exp \left(-2 \mathrm{i} s_{1}\right)} \\
& s_{1}=s_{0}+s_{d}+\pi / 2 \\
& A=\frac{\alpha_{1} / \alpha_{2}\left(z_{d} / z_{D}\right)^{1 / 2}-1}{\alpha_{1} / \alpha_{2}\left(z_{d} / z_{D}\right)^{1 / 2}+1} \exp \left[-2 \mathrm{i}\left(s_{d}+\pi / 4\right)\right] \\
& B=\frac{k\left\{1+A \exp \left[-2 \mathrm{i}\left(s_{0}+\pi / 4\right)\right]\right\}-\mathrm{i} k_{0}\left\{1-A \exp \left[-2 \mathrm{i}\left(s_{0}+\pi / 4\right)\right]\right\}}{k\left\{1+A \exp \left[-2 \mathrm{i}\left(s_{0}+\pi / 4\right)\right]\right\}+\mathrm{i} k_{0}\left\{1-A \exp \left[-2 \mathrm{i}\left(s_{0}+\pi / 4\right)\right]\right\}} \\
& k_{0}=\left[2\left(V_{0}-E\right)\right]^{1 / 2}
\end{aligned}
$$

the current density $j$ in the discussed model is given by the expression

$$
j=k|C|^{2}=k C^{*} C,
$$

where $k=(2 E)^{1 / 2}$.

\section{References}

[1] J.E. Lilienfeld, Phys. Z. 23, 506 (1922).

[2] R.H. Fowler, L. Nordheim, Proc. R. Soc. Lond. A 110, 173 (1928).

[3] A. Zener, Proc. R. Soc. Lond. A 145, 523 (1934).

[4] W. Schottky, Phys. Z. 41, 570 (1940).

[5] J. Bardeen Phys. Rev. A 138, 717 (1947).

[6] F. Flores, J.C. Duran, A. Munoz, Phys. Scr. Vol. T 10, 102 (1987).

[7] R.V. Latham, M.S. Mousa, J. Phys. D, 10, 699 (1986).

[8] M.S. Mousa, R.V. Latham, J. Phys. (France) 47, C7-139 (1986).

[9] A. Karpowicz, S. Surma, S. Głuchowski, in Proc. 9lh Seminar Surface Phys., Acta Univ. Wratislav. Mat. Fiz. Astron. 037, 229 (1986).

[10] A. Karpowicz, S. Surma, Surf. Sci. 213, 339 (1989).

[11] K. Harigaya, Y. Wada, K. Fesser, Phys. Rev. B 42, 1276 (1989).

[12] M. Rajasekaran, S. Sankar, P.C. Baynes, V. Devanathan, Phys. Rev. B 28, 4810 (1983). 
[13] M. Abramovitz, I.A. Stegun, Ilandbook of Mathematical Functions, Dover, New York 1968.

[14] S. Glucliowski, S. Surma, A. Karpowicz, in Proc. 8th Seminar Surface Phys., in Acta Univ. Wratislav. Mat. Fiz. Astron. 847, 131 (1985).

[15] L.M. Baskin, O.I. Lvov, G.N. Fursey, Phys. Status Solidi B 47, 49 (1971).

[16] L.M. Baskin, N.W. Jegorow, W.E. Pticyn, G.N. Fursey, Pis'ma Zh. Tekh. Fiz. 5, 1345 (1979).

[17] L.M. Baskin, G.N. Fursey, Proc. 13th Symp. on Discharges, Electrical Insulation in Vacuum, Paris (1988), Paris, p. 31.

[18] M. Radny, A. Karpowicz, Acla Phys. Pol. 81, 325 (1992). 